\title{
A Novel Rough-Surface Direct Wave Suppression Algorithm based on Energy Adaptive Analysis
}

\author{
Yang Yong ${ }^{1,2}$, Zhao Weigang ${ }^{2 *}$, Du Yanliang ${ }^{2}$ and Zhang Hao ${ }^{3}$ \\ ${ }^{1}$ School of Mechanical, Electronic and Control Engineering, Beijing Jiaotong University, Beijing 100044, China \\ ${ }^{2}$ The Key Laboratory for Health Monitoring and Control of Large Structures in Hebei Province, Shijiazhuang Tiedao University, \\ Shijiazhuang 050043, China \\ ${ }^{3}$ Department of Urban \& Civil Engineering, Ibaraki University, Hitachi 316-8511, Japan
}

Received 28 May 2016; Accepted 13 September 2016

\begin{abstract}
As a nondestructive testing technology, ground penetrating radar (GPR) is widely used in the detection and recognition of high-speed railway subgrade diseases. Rough-surface direct wave suppression caused by locomotive vibration and subgrade irregularity is a challenge in the GPR signal process. Flat-surface assumption makes traditional method difficult to apply directly to the rough terrain environment. To remove rough-surface direct wave and improve the signal-to-noise ratio, a new adaptive algorithm is proposed in this paper. First, according to the characteristic of electromagnetic wave propagation, the echo model of GPR was constructed, and the composition of ground radar echo signal was analyzed. Next, the eigenvalue and eigenvector of the echo signal and ground direct wave were studied. With the increase of relative permittivity, the energy eigenvector of direct wave and echo signal converged to the same vector space. On this basis, a rough-surface direct wave suppression framework based on energy feature adaptive analysis was proposed. Finally, a numerical simulation and field experiment were carried out to verify the feasibility of the algorithm. Results showed that the algorithm effectively suppresses the rough ground direct wave, and the processing result preserves the target echo more completely. Without intervention, this method can be applied in automatic direct wave suppression, which will promote the development of automatic identification technology in the detection of railway subgrade diseases.
\end{abstract}

Keywords: Rough surface, Direct wave suppression, Energy adaptive analysis, Ground penetrating radar, Subgrade diseases

\section{Introduction}

Railway subgrade presents a series of diseases caused by the repeated effects of the environment and the train load, which directly threatens traffic safety. Hence, research on developing a fast, accurate, and nondestructive disease detection method has become an urgent matter.

Ground penetrating radar (GPR) is a type of nondestructive testing technology used to detect an underground structure with the reflection characteristics of high-frequency electromagnetic waves at the interface of different electrical parameters. Owing to its fast and continuous characteristics, GPR is widely used in shallowlayer target detection, e.g., land mines [1], [2], municipal pipelines [3], and structural defects [4], [5].

Influenced by factors such as air-ground interface and anisotropic background media, a variety of clutter, including ground direct wave, multiple wave, and random noise are formed in GPR echo. Direct wave is caused by the relative permittivity difference between ground and air. Taking concrete surface as an example, the relative permittivity of concrete is $\varepsilon_{r}=9$ and the relative permittivity of air is $\varepsilon_{r}=1$. According to the Fresnel reflection formula, approximately $50 \%$ of the incident energy is reflected. The target echo energy is also generally very small; if raw data are processed

*E-mail address: zhaoweig2002@163.com

ISSN: $1791-2377$ @ 2016 Eastern Macedonia and Thrace Institute of Technology. All rights reserved. directly, the loss of target echo may occur. Thus, eliminating a direct wave with great energy is the first step in target recognition.

At present, air-coupled antenna is used instead of ground-coupled antenna to accelerate the detection speed of subgrade diseases. However, for locomotive vibration and subgrade irregularity, the antenna follows the change of train position up and down, which damages the direction of the direct wave and increases the difficulty of target recognition. Suppressing rough-surface direct wave is thus a challenge.

In this paper, from the perspective of energy, the relationship between echo signal and direct wave in the energy eigenvalue and eigenvector is discussed according to the components of the GPR echo, and a rough-surface direct wave suppression framework based on energy adaptive analysis is constructed.

\section{State of the art}

Numerous studies have been carried out on direct wave suppression, and the methods used are mainly divided into three categories: time domain method [6], frequency domain method [7], [8], [9], [10], [11], and space vector method [12], [13], [14]. The main representative of the time domain method is the mean-subtraction method. With the direct wave as a constant and the target echo as the disturbance signal, this method eliminates the constant by subtracting 
between data in A-Scan and average data in B-Scan. In a frequency domain, frequency wavenumber method (F-K) and directional wavelet method are included. Wavelet method has become a hot spot in the field of direct wave suppression in recent years because of its good direction. Shi et al. [7], and Zhang et al. [8] constructed a 2D physical wavelet, which suppresses the direct wave via the different scale between direct wave and target echo. Tzanis [9], Baili et al. [10], and Zhang et al. [11] studied the direction of the direct wave and found that it changed slightly along the survey line, an outcome that is consistent with the characteristic of shearlet and ridgelet. Time domain and frequency domain methods are mainly used to remove direct wave from the morphological point of view. These methods work when the ground is flat, but they have no effect on the rough surface and even result in target echo signal loss. Abujarad et al. [12], $\mathrm{Lu}$ et al. [13], and Huo et al. [14] investigated the space vector method, e.g., principal component analysis (PCA), to eliminate direct waves. They believed that a large proportion of the direct wave leads to a large eigenvalue in PCA. Removing the main eigenvalues could eliminate the direct wave. This method does not discuss the relationship in eigenvalue between the direct wave and the GPR echo signal. Moreover, the choice of removed eigenvalue in the method is subjective, which is not conducive to realizing the automatic suppression of direct waves.

The above analysis shows that the existing methods are not applicable for suppressing rough-surface direct waves. Therefore, theoretical derivations, a forward simulation, and a field test are combined to propose a new algorithm that can suppress rough-surface direct waves.

The rest of this paper is organized as follows. In section 3, a GPR echo signal model is built, the relationship of eigenvalue and eigenvector between GPR echo signal and direct wave is analyzed, and then an energy-adaptive-based algorithm is proposed. In Section 4, forward simulation and field test are carried out to verify the feasibility of the proposed method. In Section 5, signal loss in different methods is discussed. In Section 6, the article is summarized and related conclusions are drawn.

\section{Methodology}

\subsection{GPR echo signal model}

Let us consider a common-offset GPR [21]. When the highfrequency electromagnetic waves emitted by the transmitter encountered the air-ground interface, the reflected waves were collected by the receiver, while the transmitted waves continued to spread. Part of the transmitted waves would also be reflected at the surface of the target with different relative permittivities and go through the air-ground surface to be collected. Fig. 1 shows a schematic model of GPR echo signal. In the figure, $\mathrm{T}$ represents the transmitter, $\mathrm{R}$ represents the receiver, $S$ represents the GPR echo signal, $D$ represents the direct wave, $O$ represents the target echo, and $n$ represents the random noise. Thus, the GPR echo signal can be expressed as:

$$
X=D+O+n
$$

The parameters of the air and ground are $\left(\varepsilon_{r 1}, \mu_{r 1}, \sigma_{1}\right)$ and $\left(\varepsilon_{r 2}, \mu_{r 2}, \sigma_{2}\right)$ respectively, where $\varepsilon_{r}$ represents relative permittivity, $\mu_{r}$ represents relative permeability, and $\sigma$ represents conductivity.

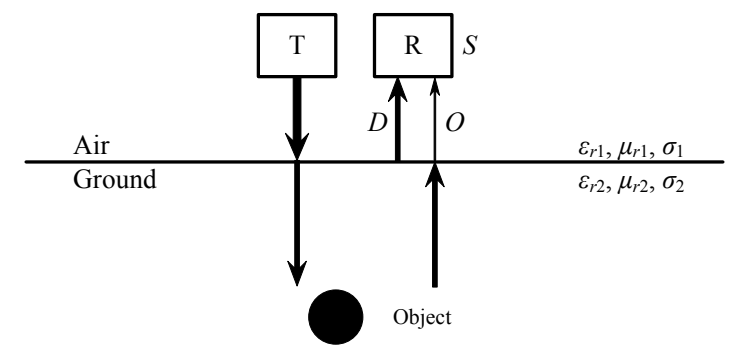

Fig. 1. Schematic model of the GPR echo signal

Working with the hypothesis that the GPR surveys $l$ times along the line, single-measurement data $S_{i}$, e.g., the $i$ th measurement, is called the A-Scan, $S_{i}=\left(s_{i l}, s_{i 2}, \cdots, s_{i k}\right)^{T}$, where $k$ represents the sampling points.

All of the A-Scans make up the B-Scan, which can be expressed as Matrix $S$ :

$$
S=\left[S_{1}, S_{2}, \mathrm{~L}, S_{n}\right]=\left[\begin{array}{llll}
s_{11} & s_{21} & \mathrm{~L} & s_{l 1} \\
s_{12} & s_{22} & \mathrm{~L} & s_{l 3} \\
\mathrm{~L} & \mathrm{~L} & \mathrm{~L} & \mathrm{~L} \\
s_{1 k} & s_{2 k} & \mathrm{~L} & s_{l k}
\end{array}\right]
$$

Similar to the GPR echo signal, the direct-wave matrix $D$ can be expressed as:

$$
D=\left[D_{1}, D_{2}, \mathrm{~L}, D_{n}\right]=\left[\begin{array}{llll}
d_{11} & d_{21} & \mathrm{~L} & d_{l 1} \\
d_{12} & d_{22} & \mathrm{~L} & d_{l 3} \\
\mathrm{~L} & \mathrm{~L} & \mathrm{~L} & \mathrm{~L} \\
d_{1 k} & d_{2 k} & \mathrm{~L} & d_{l k}
\end{array}\right]
$$

\subsection{Energy characteristics of echo signal}

The correlation operation is developed to obtain the energy characteristic of the echo signal. Consider $c_{m n}$ as the correlation between the $m$ th trace data and the $n$th trace data, i.e.:

$$
c_{m n}=\sum_{j=1}^{k} s_{m j} s_{n j}
$$

The correlation matrix $C$, which is composed of $c_{m n}$, can be expressed as:

$$
C=\left[\begin{array}{llll}
c_{11} & c_{12} & \mathrm{~L} & c_{1 l} \\
c_{21} & c_{22} & \mathrm{~L} & c_{2 l} \\
\mathrm{~L} & \mathrm{~L} & \mathrm{~L} & \mathrm{~L} \\
c_{l 1} & c_{l 2} & \mathrm{~L} & c_{l l}
\end{array}\right]
$$

where $c_{m n}=c_{n m}$, which means matrix $C$ is a real symmetric matrix.

And there must be a standard orthogonal matrix $P$, which is satisfied as $P^{-1} C P=\Lambda$ and $P^{-1}=P^{T}$. The superscript $T$ denotes transpose, and $\Lambda$ denotes the diagonal matrix:

$\Lambda=\operatorname{diag}\left(\lambda_{1}, \lambda_{2}, \mathrm{~L}, \lambda_{l}\right)$ 
Values $\left(\lambda_{1}, \lambda_{2}, \cdots, \lambda_{l}\right)$ are the eigenvalues of correlation matrix $C$, and the standard orthogonal matrix $P$ is the matrix consisting of eigenvectors.

Given that the vectors in the standard orthogonal matrix are not related to one another, then inner product $r_{i j}$ between eigenvector $P_{i}$ and $P_{j}$ can be written as:

$$
r_{i j}=\left(P_{i}, P_{j}\right)= \begin{cases}1 & i=j \\ 0 & i \neq j\end{cases}
$$

Therefore, matrix $P$ can be regarded as an orthonormal basis in $l$-dimensional space, and the projection of GPR echo signal data $S$ on $P$ can be expressed as:

$$
S^{\prime}=P^{T} S
$$

The reconstruction formula is

$$
S=P S^{\prime}
$$

Meanwhile, the sum of eigenvalues in Eq. (5) is equal to that of the diagonal elements in matrix $C$, which is

$$
\begin{aligned}
\operatorname{trace}(C) & =c_{11}+c_{22}+\mathrm{L}+c_{l l} \\
& =\lambda_{1}+\lambda_{2}+\mathrm{L}+\lambda_{l}
\end{aligned}
$$

In addition, with a descending order, let $\lambda_{1}>\lambda_{2}>\cdots>\lambda_{l}$, where $\lambda_{i}$ denotes the energy characteristics of the GPR echo signal at base $P_{i}$.

\subsection{Energy characteristic of direct wave}

Assuming that the GPR echo signal is a finite-length signal, no overlapped signal exists between direct wave and target echo, and nonzero value ranges of the direct wave is $j \in[1$, $b], b<l$. Thus, the direct wave value can be expressed as

$d_{i j}=\left\{\begin{array}{rl}s_{i j} & j \in[1, b] \\ 0 & \text { others }\end{array}\right.$

where

$D=\left[D_{1}, D_{2}, \mathrm{~L}, D_{n}\right]=\left[\begin{array}{llll}d_{11} & d_{21} & \mathrm{~L} & d_{l 1} \\ d_{12} & d_{22} & \mathrm{~L} & d_{l 3} \\ \mathrm{~L} & \mathrm{~L} & \mathrm{~L} & \mathrm{~L} \\ d_{1 k} & d_{2 k} & \mathrm{~L} & d_{l k}\end{array}\right]$

Similar to the details in Section 3.2, eigenvalue $\lambda_{i}{ }^{\prime}$ and eigenvector $P_{i}^{\prime}$ were obtained according to the correlation matrix of $D$. Furthermore, with a descending order, let $\lambda_{1}{ }^{\prime}>\lambda_{2}{ }^{\prime}>\ldots>\lambda_{l}$, where $\lambda_{i}{ }^{\prime}$ denotes the energy characteristics of direct wave at base $P_{i}^{\prime}$.

\subsection{Energy adaptive reconstruction}

Under the condition that the GPR echo signal contains no target echo signal, or the permittivity of the target is very close to that of the background medium which means target echo energy is very low, the equations $D=S$ and $P_{i}{ }^{\prime}=P_{i}$ are set up. Given that $P$ is an orthogonal matrix, the inner product $r_{i j}{ }^{\prime}$ between $P_{i}{ }^{\prime}$ and $P_{j}$ can be written as:

$$
r_{i j}{ }^{\prime}=\left(P_{i}^{\prime}, P_{j}\right) \approx \begin{cases}1 & i=j \\ 0 & i \neq j\end{cases}
$$

Hence, eigenvector $\lambda_{i}{ }^{\prime}$ denotes the energy characteristics of direct wave at base $P_{i}$. As the total energy is $\lambda_{i}$ at the base $P_{i}$, to eliminate the direct wave, base $P_{i}$ is modified to $P_{i}{ }^{\prime \prime}$ :

$P_{i}^{\prime \prime}=P_{i} \frac{\left|\sqrt{\lambda_{i}}-\sqrt{\lambda_{i}}\right|}{\sqrt{\lambda_{i}}}$

Then reconstructing the GPR echo signal $S^{\prime \prime}$ can be expressed as:

$S^{\prime \prime}=P^{\prime \prime} S^{\prime}$

where $S^{\prime \prime}$ does not contain direct wave any more. Fig. 2 describes the flow chart of the algorithm.

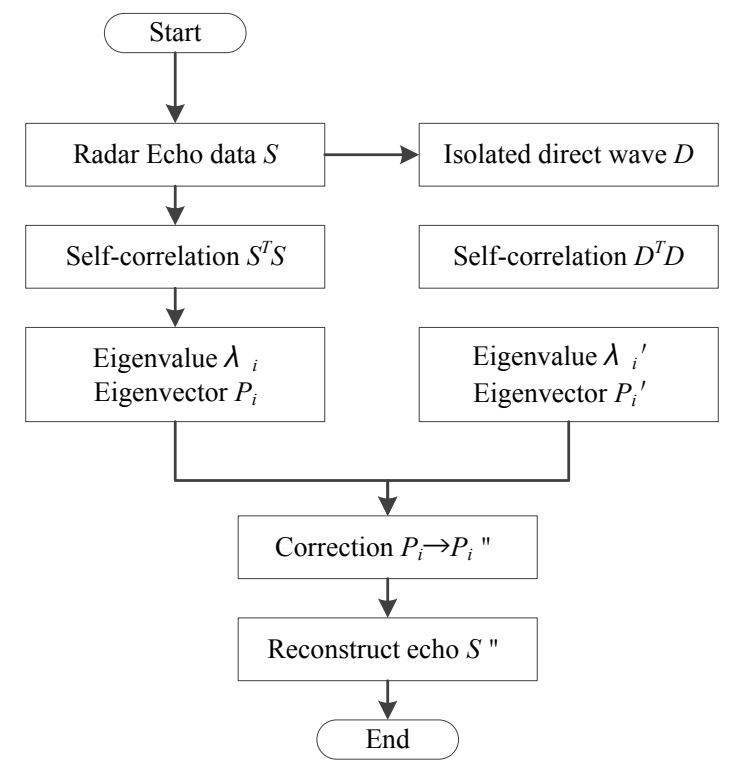

Fig. 2. Flow chart of energy adaptive algorithm

\section{Forward simulation and field test}

To verify the feasibility of the proposed method, forward modeling simulation and field test are carried out.

\subsection{Forward modeling}

Forward modeling is designed to verify the relationship between GPR echo signal and direct wave in the energy eigenvector and eigenvalue. The model is established by GprMax [15], [16], [17], as shown in Fig. 3. The modeling method is based on finite-difference time-domain [18], [19], [20], [21]. The modeling parameters are shown in Tab. 1.

The model's dimension is $2.0 \mathrm{~m} \times 0.6 \mathrm{~m}$ and is divided into two layers. The upper layer is free space with parameters $\left(\varepsilon_{r 1}, \mu_{r 1}, \sigma_{1}\right)=(1,1,0)$, and the lower layer is the background medium $\left(\varepsilon_{r 2}, \mu_{r 2}, \sigma_{2}\right)=\left(\varepsilon_{r 2}, 1,0.01\right)$. The target is a good conductor rebar. To check the correlation between $P_{i}{ }^{\prime}$ and $P_{i}$ of various relative permittivities, set the range of $\varepsilon_{r 2} \in[4,16]$. A tilted surface likewise exists, where $x \in[0.5$, 1.5] with an angle $\theta=5.7^{\circ}$.

Fig. 4 demonstrates the forward simulation results where $\varepsilon_{r 2}=\{4,9,16\}$. The direct wave is not in a line. Directionbased methods, e.g., mean-subtraction method and 
directional wavelet method, have no effect on direct wave removal. "A" in the figure represents the target echo. For the wave velocity inversely proportional to the square root of relative permittivity, the two-way travel time of the target echo is shortest when $\varepsilon_{r 2}=4$, longer when $\varepsilon_{r 2}=9$, and the longest when $\varepsilon_{r 2}=16$.

Fig. 5 shows the energy comparison between direct wave and target echo in different media. In Fig. 5(a), direct wave and target echo are pointed out under the condition of $\varepsilon_{r 2}=4$ The amplitude of direct wave is distinctly greater than that of the target echo, which is consistent with the large energy assumption of the direct wave. Fig. 5(b) shows the amplitude of direct wave and the target echo in different relative permittivities. Regularity reveals that the amplitude of direct wave increases and that of the target echo decreases with increasing background relative permittivity $\varepsilon_{r 2}$.

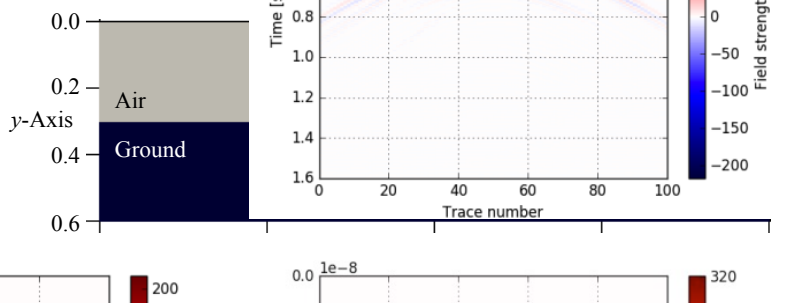

Fig. 3. Forward simulation modeling

Table 1. Forward modeling parameters

\begin{tabular}{c|c|c|c}
\hline Parameter & Values & Parameter & Values \\
\hline Model size $(\mathrm{m}, \mathrm{m})$ & $(2.0,0.6)$ & Antenna frequency $(\mathrm{Hz})$ & $2 \mathrm{G}$ \\
$\Delta x(\mathrm{~m})$ & 0.004 & Excitation & Ricker \\
$\Delta z(\mathrm{~m})$ & 0.004 & Samples & 1236 \\
Time window $(\mathrm{ns})$ & 16 & Target radius $(\mathrm{m})$ & 0.01 \\
\hline
\end{tabular}
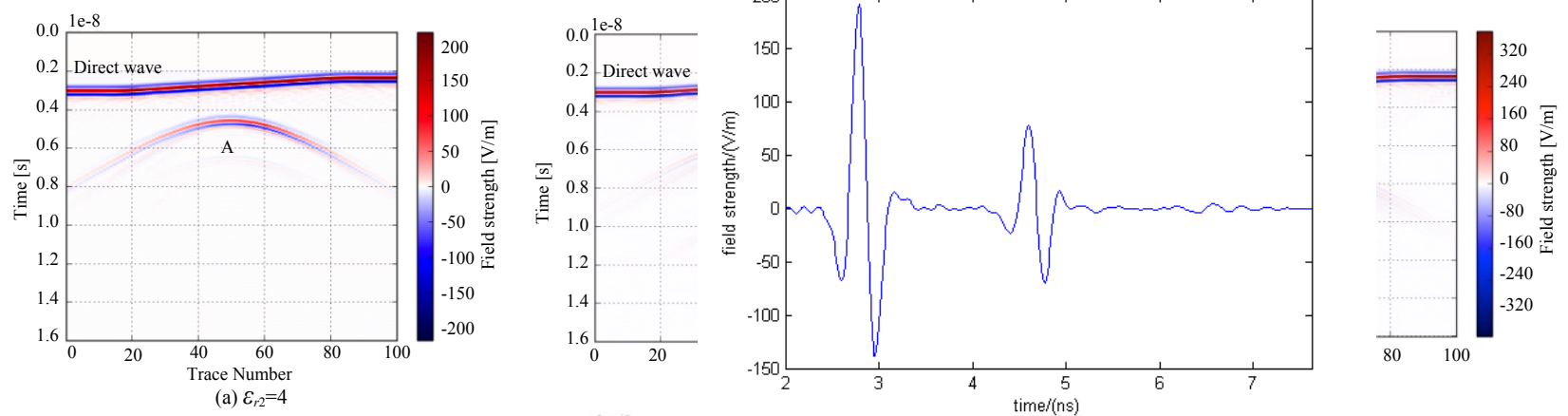

Fig. 4. Forward simulation result
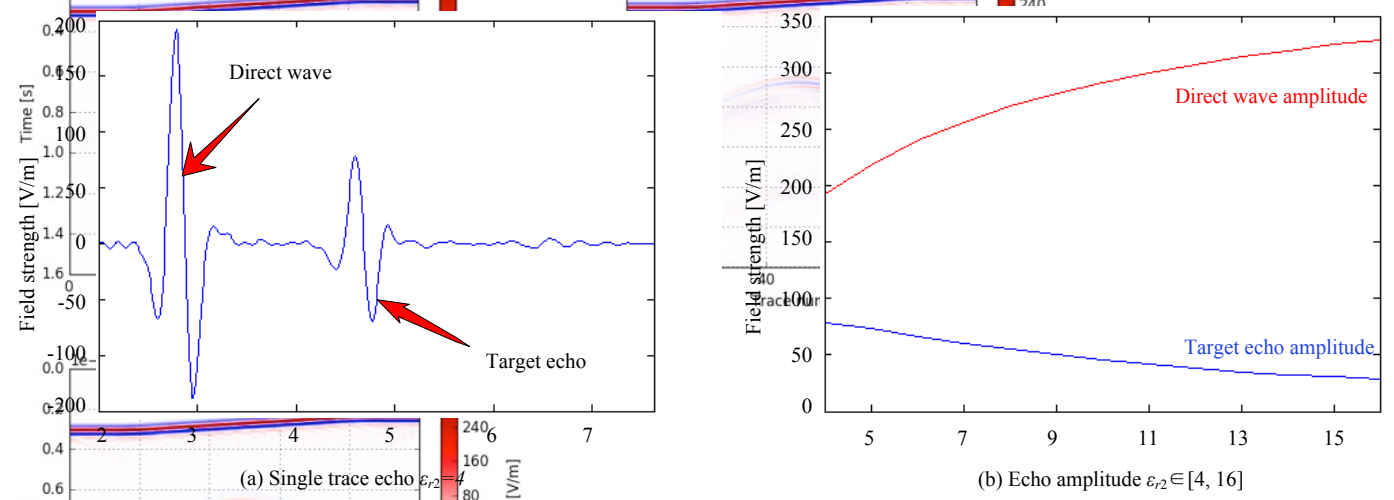

Fig. 5. Energy comparisons between direct wave and target echo

The simulation results are processed according to the proposed method and processing flow. Tab. 2 lists the largest normalized eigenvalues from $\lambda_{1}$ to $\lambda_{6}$. Tab. 3 shows the correlation between $P_{i}$ and $P_{i}{ }^{\prime}$ in different background media.

Table. 2. Eigenvalue comparison in different media

\begin{tabular}{c|c|c|c|c|c|c}
\hline \multirow{2}{*}{} & \multicolumn{2}{|c|}{$\boldsymbol{\varepsilon}_{\boldsymbol{r} 2}=\mathbf{4}$} & \multicolumn{2}{c|}{$\boldsymbol{\varepsilon}_{\boldsymbol{r} 2}=\mathbf{9}$} & \multicolumn{2}{c}{$\boldsymbol{\varepsilon}_{\boldsymbol{r} 2}=\mathbf{1 6}$} \\
\cline { 2 - 7 } & $\boldsymbol{S}$ & $\boldsymbol{D}$ & $\boldsymbol{S}$ & $\boldsymbol{D}$ & $\boldsymbol{S}$ & $\boldsymbol{D}$ \\
\hline$\lambda_{1}$ & 1 & 0.997 & 1 & 0.999 & 1 & 1 \\
$\lambda_{2}$ & 0.721 & 0.714 & 0.707 & 0.705 & 0.703 & 0.702 \\
$\lambda_{3}$ & 0.233 & 0.232 & 0.227 & 0.227 & 0.225 & 0.225 \\
$\lambda_{4}$ & 0.043 & 0.037 & 0.037 & 0.036 & 0.036 & 0.036 \\
$\lambda_{5}$ & 0.013 & 0.003 & 0.004 & 0.003 & 0.003 & 0.003 \\
$\lambda_{6}$ & 0.010 & 0.002 & 0.002 & 0.001 & 0.001 & 0.001 \\
\hline
\end{tabular}

Table. 3. Eigenvector correlation in different media

\begin{tabular}{c|c|c|c|c}
\hline$\varepsilon_{r 2}$ & $\left(P_{1}, P_{1}^{\prime}\right)$ & $\left(P_{2}, P_{2}^{\prime}\right)$ & $\left(P_{3}, P_{3}^{\prime}\right)$ & $\left(P_{4}, P_{4}{ }^{\prime}\right)$ \\
\hline 4 & 0.999856 & 0.998879 & 0.977721 & 0.998423 \\
9 & 0.999888 & 0.99924 & 0.993687 & 0.999463 \\
16 & 0.999897 & 0.99929 & 0.997554 & 0.999664 \\
\hline
\end{tabular}

Tab. 2 and Tab. 3 show that a good energy correlation in eigenvalues and eigenvectors exists between direct wave and target echo; the correlation also increases with the increase of background relative permittivity. This correlation would make the eigenvectors converge to the same eigenvector space.

Fig. 6 is the processing result based on energy adaptive analysis, where $\varepsilon_{r 2}=\{4,9,16\}$.

A comparison of Figs. 4 and Fig. 6 clearly indicates that the direct wave in Fig. 4 is suppressed. Specifically, with the increase of background relative permittivity, the effect is more significant. From Fig. 6(c), under the condition of $\varepsilon_{r 2}=16$, the direct wave is removed, and the target echo hyperbola is very clear and preserved completely without break. This finding means that the energy-adaptive-based method can suppress the direct wave efficiently, and with the increase of relative permittivity, the effect is improved performance. Considering that relative permittivity of reinforced concrete in a humid environment generally is 
bigger than 8 , this method can be used to suppress direct wave in high-speed railways.

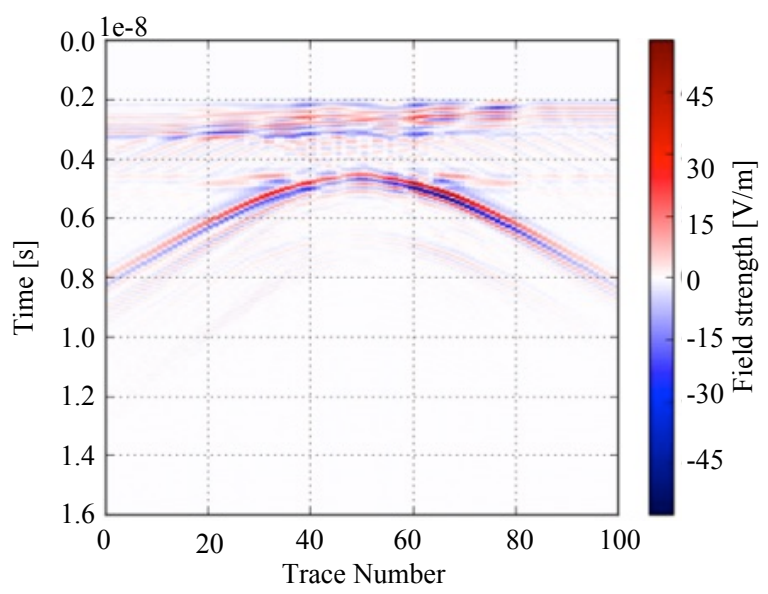

(a) $\varepsilon_{r 2}=4$

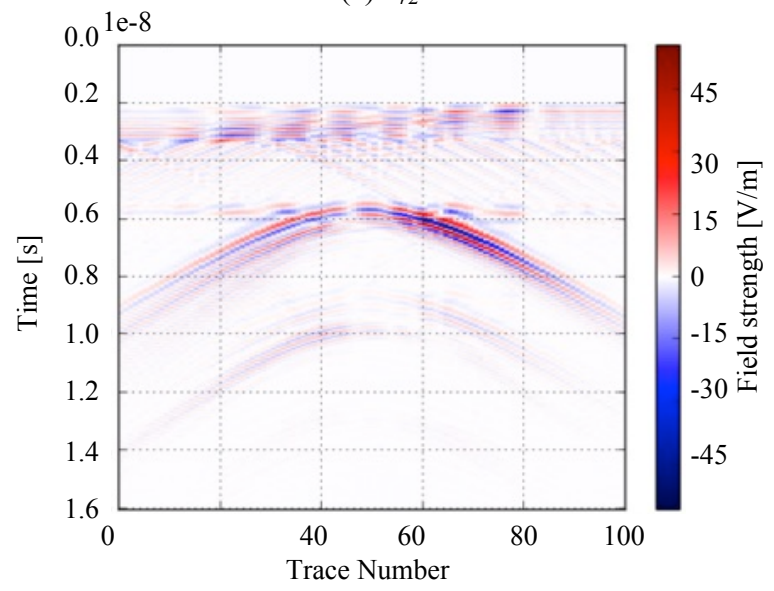

(b) $\varepsilon_{r 2}=9$

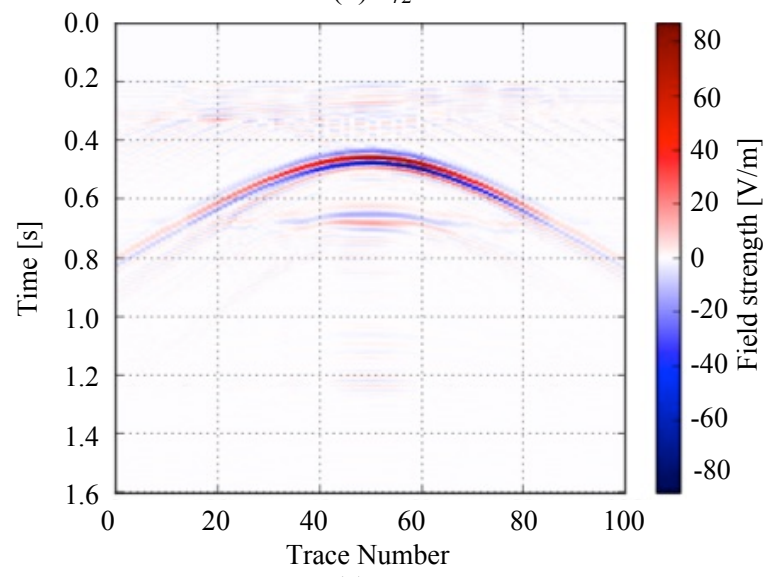

(c) $\varepsilon_{r 2}=16$

Fig. 6. Processing result in different media

\subsection{Field experiment}

A field experiment is designed to verify the availability in practice. The CRTS-II slab ballastless track laying in the training center of Shijiazhuang Tiedao University provided the experimental subject, and IDS-RIS GPR host and $2 \mathrm{GHz}$ antenna were used to acquire data. The test field image is shown in Fig .7.

CRTS-II slab ballastless track is made up of slab, CA mortar, and a support layer. The slab layers are reinforced concrete with a "V"-type water sink in interval $d=0.65$, which means it is not flat. The GPR parameters are set as shown in Tab. 4.

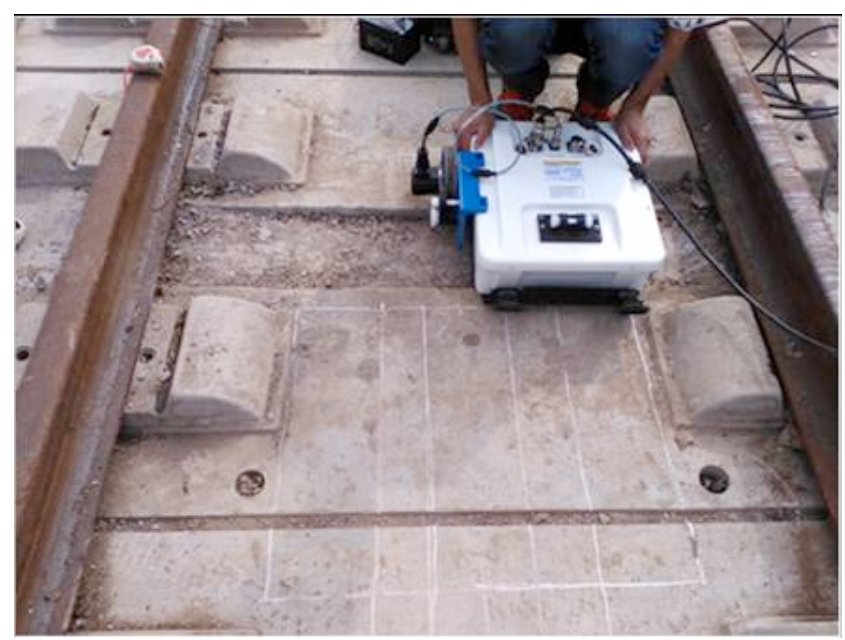

Fig. 7. Field test with CRTS-II slab ballastless track

Table 4. Field test sampling parameters

\begin{tabular}{c|c|c|c}
$\begin{array}{c}\text { Frequency } \\
\text { (MHz) }\end{array}$ & Sample points & Window (ns) & $\begin{array}{c}\text { Interval } \\
(\mathbf{m m})\end{array}$ \\
\hline 900 & 512 & $40 \mathrm{~ns}$ & 8 \\
\hline
\end{tabular}

Fig. 8(a) shows a part of the acquired data. Directional characteristic of the direct wave is damaged while water sink echoes are introduced. Fig. 8(b) shows processing result according to the proposed method. The ground direct wave is found to be almost eliminated, and the echo of the reinforced bar is clearly visible, which verifies the feasibility of the algorithm in the practical application.

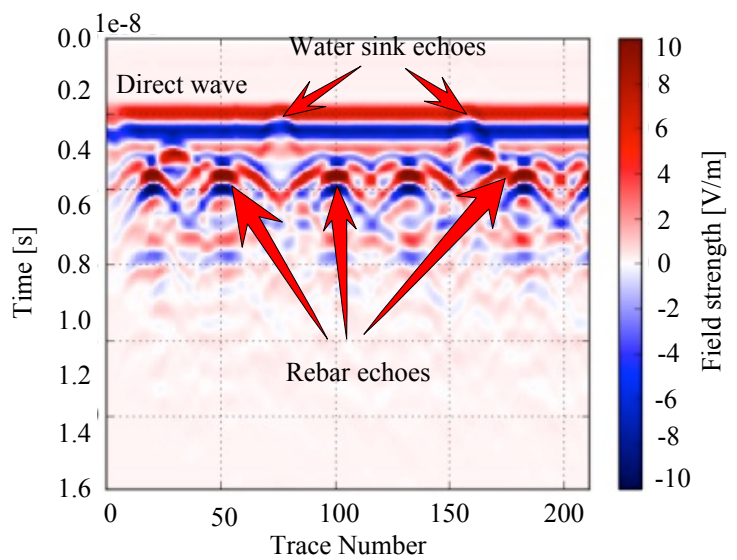

(a) Original data in test field

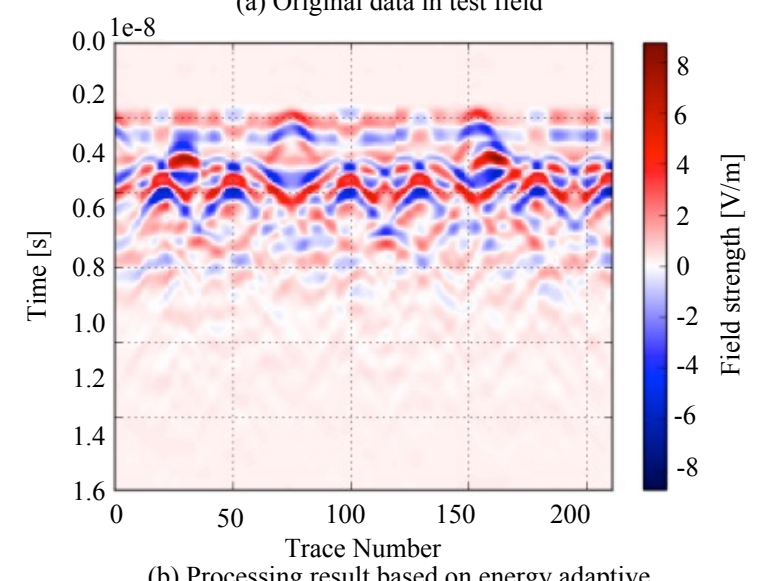

(b) Processing result based on energy adaptive

Fig. 8. Original data and processing result 


\section{Discussion}

The purpose of suppressing noise is to improve signal-tonoise ratio (SNR). Given that the mean-subtraction method and directional wavelet method are unable to eliminate the direct wave rough ground, SNR discussions are carried out on the processing results based on PCA and the proposed energy adaptive method.

The original data obtained by field experiment are shown in Fig. 8(a). Fig. 9 shows the processed result by PCA, which moves the largest two eigenvalues and corresponding eigenvectors.

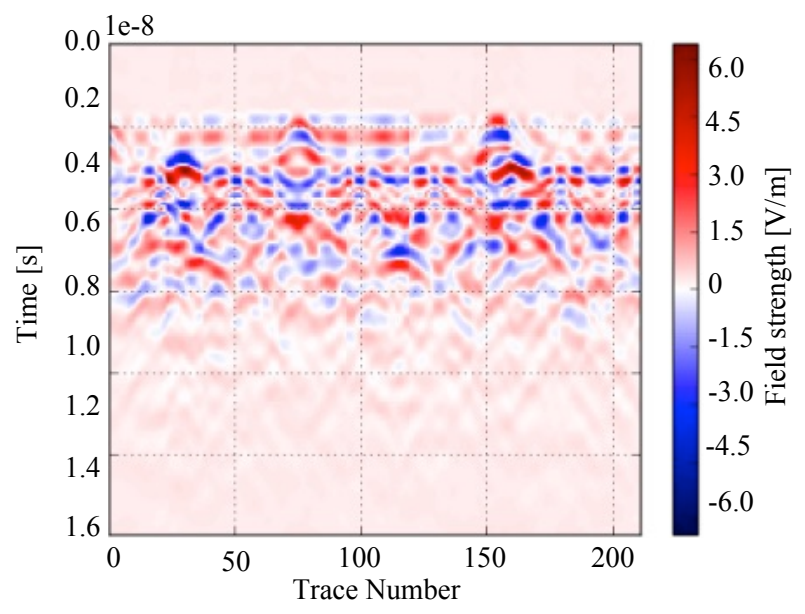

Fig. 9. Processing result based on PCA

Compared with the details in Fig. 8(b), the maximum amplitude in Fig. 9 is less than that of the energy-adaptiveprocessed data. This result means the PCA-based method will result in more signal loss.

To analyze the target echo signal loss, correlation coefficient $r$ and average cumulative error $\Delta$ are introduced to evaluate the integrity of the signal; they are calculated as Eq. (13) and Eq. (14) below:

$$
r=\frac{\sum\left(x_{i}-\bar{x}\right)\left(y_{i}-\bar{y}\right)}{\sqrt{\sum\left(y_{i}-\bar{y}\right)^{2}} \sqrt{\sum\left(y_{i}-\bar{y}\right)^{2}}}
$$

$$
\Delta=\frac{1}{k} \sum\left|x_{i}-y_{i}\right|
$$

where $x_{i}$ donates the processed data, $y_{i}$ donates the original data, $\bar{x}$ is the average of $x_{i}$, and $\bar{y}$ is the average of $y_{i}$.

The traces of $i=\{25,50,75,100,125,150\}$ are extracted from original data, PCA processed data, and energy adaptive processed data, and processed according to Eq. (13) and Eq. (14). The results are shown in Tab. 5.

Results indicate that the energy-adaptive-based method has better performance than the PCA-based method in correlation coefficient and average cumulative error. Thus, the energy-adaptive-based method can be used as an efficient way to suppress direct waves.

Table. 5. Correlation coefficient and error comparisons

\begin{tabular}{c|c|c|c|c}
\hline \multirow{2}{*}{$\begin{array}{c}\text { Trace } \\
\text { number }\end{array}$} & \multicolumn{2}{|c|}{ PCA based } & \multicolumn{2}{c}{ Energy adaptive based } \\
\cline { 2 - 5 } & $r$ & $\Delta$ & $r$ & $\Delta$ \\
\hline 25 & 0.85 & 0.29 & 0.91 & 0.07 \\
50 & 0.91 & 0.38 & 0.94 & 0.25 \\
75 & 0.72 & 0.31 & 0.82 & 0.10 \\
100 & 0.64 & 0.33 & 0.78 & 0.11 \\
125 & 0.83 & 0.29 & 0.90 & 0.07 \\
150 & 0.85 & 0.39 & 0.95 & 0.22 \\
\hline Average & 0.80 & 0.33 & 0.88 & 0.14 \\
\hline
\end{tabular}

\section{Conclusion}

In this study, the relationship between echo signal and direct wave in the energy eigenvalue and eigenvector is discussed, and a new energy-adaptive-based algorithm is proposed to suppress direct waves. The conclusion are as follows:

(1) The relationship between direct wave and GPR echo indicates that the energy eigenvector converges to the same space with the increase of background medium relative permittivity.

(2) The method does not depend on the direction of the direct wave and can effectively eliminate rough-surface direct wave in the perspective of energy eigenvalue and eigenvector. Therefore, it overcomes the shortcomings of the mean-subtraction method and the directional wavelet method. Additionally, the effect will be better with the increase of background medium relative permittivity.

(3) Numerical simulation and experimental results show that compared with the PCA method, the processed data based on energy adaptive method is more effective in retaining the target echo signal and has a higher SNR with the correlation coefficient of 0.88 .

With huge data quantity in detecting railway subgrade diseases, the disadvantages of artificial identification, such as long period and high false alarm rate, are displayed. The proposed method is a reliable and suitable way to suppress direct wave automatically, which will promote the development of automatic identification technology in the detection of railway subgrade diseases.

\section{Acknowledgement}

This study was financially supported by National Natural Science Foundation of China (No. 51578349) and the Railway Ministry of Science and Technology Research and Development Program of China (No. 2013G004-A-1).

\section{References}

1. Smitha N., Bharadwaj D. R. U., Abilash S., et al, "Kirchhoff and F-K migration to focus ground penetrating radar images". International Journal of Geo-Engineering, 7(1), 2016, pp.1-12.

2. Missaoui O., Frigui H., Gader P., "Land-Mine detection with Ground-Penetrating Radar using Multistream Discrete Hidden Markov Models". Geoscience \& Remote Sensing IEEE Transactions on, 49(6), 2011, pp.2080-2099.

3. Seyfried D., Jansen R., Schoebel J., "Shielded loaded bowtie antenna incorporating the presence of paving structure for improved GPR pipe detection". Journal of Applied Geophysics, 111, 2014, pp.289-298.
4. Lalagüe A., Lebens M. A., Hoff I., "Detection of Rockfall on a tunnel concrete jining with Ground-Penetrating Radar (GPR)". Rock Mechanics \& Rock Engineering, 49(7), 2016, pp.2811-2833.

5. Xisheng D., Tian D., Quan Y., "Tunnel lining thickness and voids detection by GPR". Electronic Journal of Geotechnical Engineering, 20(7), 2015, pp.2019-2030.

6. Brunzell H., "Detection of shallowly buried objects using impulse radar". IEEE Transactions on geoscience and remote sensing, 37(2), 1993, pp.875-886. 
Yang Yong, Zhao Weigang, Du Yanliang and Zhang Hao/

Journal of Engineering Science and Technology Review 9 (4) (2016) 202 - 208

7. Shi X., Yang Q., "Suppressing the direct wave noise in GPR data via the 2-D physical wavelet frame". International Conference on Transportation, Mechanical, and Electrical Engineering, Changchun, China: TMEE, 2011, pp.1161-1164.

8. Zhang R. F., Tadeusz J., "Ultych. Physical wavelet frame denoising". Goephysic, 68 (1), 2003, pp.225- 231.

9. Tzanis A., "Detection and extraction of orientation-and-scaledependent information from two-dimensional GPR data with tuneable directional wavelet filters". Journal of Applied Geophysics, 89, 2013, pp.48-67.

10. Baili J., Lahouar S., Hergli M., "GPR signal de-noising by discrete wavelet transform”, $N d t \&$ E International, 42(8), 2009, pp.696-703.

11. Zhang Z. Y., Zhang J. L., Yu H. Y., "Ridgelet transform with application in ground penetrating radar processing". International Conference on Wavelet Analysis and Pattern Recognition. Beijing, China: IEEE, 2007, pp.1054 - 1059.

12. Abujarad F., Omar A., "GPR Data Processing Using the Component-Separation Methods PCA and ICA". International Workshop on Imagining Systems and Techniques, Minori, Italy: IEEE, 2006, pp.60-64.

13. Lu Q. Q., Pu J. X., Wang X. H., "A Clutter Suppression Algorithm for GPR Data Based on PCA Combining with Gradient Magnitude". Applied Mechanics \& Materials, 2014, pp.1662-1667.

14. Huo Z. H., Wang M. H., "The Application of KL Transform to Remove Direct Wave in Ground Penetrating Radar Records". International Conference on Image and Graphics. Sichuan, China: IEEE, 2007, pp.133-138.
15. Warren C., Giannopoulos A., Giannakis I., "An advanced GPR modelling framework: The next generation of gprMax". International Workshop on Advanced Ground Penetrating Radar. Florence, Italy : IEEE, 2015, pp.1-4.

16. Giannakis, I., Giannopoulos A., Warren, C.. "A Realistic FDTD Numerical Modeling Framework of Ground Penetrating Radar for Landmine Detection". IEEE Journal of Selected Topics in Applied Earth Observations and Remote Sensing, 9(1), 2016, pp.37-51.

17. Giannopoulos, A., "Modelling ground penetrating radar by GprMax", Construction and Building Materials, 19(10),2005, pp.755762

18. Yee K. S., Ingham D., Shlager K., "Time-domain extrapolation to the far field based on FDTD calculations". IEEE Transactions on Antennas \& Propagation, 39(3), 1991, pp.410-413.

19. Shangguan P., Al-Qadi I. L., "Calibration of FDTD Simulation of GPR Signal for Asphalt Pavement Compaction Monitoring". IEEE Transactions on Geoscience \& Remote Sensing, 53(3), 2015, pp.15381548 .

20. Bérenger J. P., "On the Huygens absorbing boundary conditions for electromagnetics". Journal of Computational Physics, 226(1), 2007, pp.354-378.

21. Lai W. L., Kind T., Sham F. C., "Correction of GPR wave velocity at different oblique angles between traverses and alignment of line objects in a common offset antenna setting". Ndt \& E International, 82, 2016, pp.36-43. 